\title{
Micro Fluxgate Sensor using Solenoid Coils Fabricated by MEMS Technology
}

\author{
Jian Lei, Chong Lei, Yong Zhou* \\ Science and Technology on Micro/Nano Fabrication Laboratory, Shanghai Jiao Tong University, \\ Dongchuan Road 800, Shanghai 200240, China, yzhou@sjtu.edu.cn*
}

In this paper, we present a micro fluxgate sensor with solenoid coils. Micro-solenoid coils were used in this fluxgate sensor as excitation and sensing elements. Two identical excitation coils in inverse series were wound around the opposite sides of the rectangular-ring-shaped magnetic core, respectively. The sensing coil was placed vertically to the excitation coils and between them. Adopting gluing and chemical wet etching, iron-based nanocrystalline alloy was used as magnetic core material. This fluxgate sensor was designed to operate based on second harmonic operation principle. $100 \mathrm{kHz}$ excitation currents with different effective values were selected to characterize the fabricated fluxgate sensor. From these experiments, we obtained some results from which we found out that the greatest sensitivity of $583.1 \mathrm{~V} / \mathrm{T}$ was achieved with the effective excitation current of $150 \mathrm{~mA}$. In the range from $-500 \mu \mathrm{T}$ to $+500 \mu \mathrm{T}$, the fabricated fluxgate sensor can keep a good linearity. The noise power density was 13.57 $n T / \mathrm{Hz}^{0.5}$ at $1 \mathrm{~Hz}$ and the noise RMS level was $23 \mathrm{nT}$ in the frequency range of $10 \mathrm{mHz}-25 \mathrm{~Hz}$.

Keywords: Fluxgate sensor, magnetic sensor, micro-solenoid coil, nanocrystalline alloy, magnetic material

\section{INTRODUCTION}

$\mathrm{M}$ AGNETIC FIELD sensing and measuring has always been an essential function in many applications for years [1]. One of the most important sensitive magnetic sensors is the fluxgate magnetic sensor [2-4]. Fluxgate sensor measures DC or low-frequency AC magnetic fields and possesses many advantages over the other magnetic sensors. In terms of resolution of the sensors, fluxgate sensor resolution is higher than the other solid-state devices such as Hall effect and magnetostrictive sensors, and is comparable to ultrahigh sensitive but very expensive quantum-effect SQUIDs [5, 6]. Fluxgate magnetic sensor can be used in many applications, such as geophysics, space research [7] and so on.

In recent trends, fluxgate sensor is more and more urgently demanded to be smaller in size, lighter in weight, lower in power consumption and more suitable for integration of the supporting electronic circuitry. Although there are some difficulties in the micromachining of fluxgate sensors with tridimensional solenoid coils, this kind of fluxgate sensors has smaller dimension and lower noise than those with planar coils as solenoid coils for the ideal coupling between tridimensional coils and magnetic cores. Thus, tridimensional coils used in micro fluxgate sensors could be the researching focus at present and in the future for particular applications such as magnet activated switches for cellular phones, inclinometer for petroleum exploration and so on.

For the material of which the fluxgate sensor core is made, we selected iron-based nanocrystalline alloy rather than permalloy traditionally used for fluxgate magnetic cores. Iron-based nanocrystalline alloy possesses high saturation induction density which will increase the measuring range of fluxgate sensors. Moreover, high permeability can lead to large inductance, and high resistivity can reduce eddy current loss. So, using an iron-based nanocrystalline alloy ribbon as a magnetic material can further improve the measuring sensitivity of the sensor and decrease the power consumption.

Recently, fluxgate sensors with different structures and core materials have been developed. A compact planar fluxgate sensor using a ferromagnetic amorphous metal core is described in [8]. Zorlu and Kejik presented a microfabricated orthogonal fluxgate sensor with electroplated permalloy core [9].

In this work, a micro fluxgate sensor with tridimensional solenoid coils acting as excitation and sensing elements and a magnetic core made of iron-based nanocrystalline alloy is designed, fabricated, and characterized.

\section{FABRICATION}

Fabrication of the micro fluxgate sensor by the MEMS technique has been mature with continual improvement $[10$, 11]. In this work, gluing and chemical wet etching was used to fabricate the magnetic core. Thick photoresist-based UV lithography was adopted in the fabrication of the solenoid coils. The fabrication steps of the fluxgate sensor are summarized in Fig. 1 and described as follows:

(1) $\mathrm{A} \mathrm{Cr} / \mathrm{Cu}$ seed layer was deposited on the wafer and a photoresist layer was spun on the seed layer. Using the photolithography technology, photoresist was patterned to obtain molds in which the bottom parts of the coils were electroplated. The thickness of the coil lines can be determined by the thickness of the spun photoresist. In this work, the thickness of the coil lines was $20 \mu \mathrm{m}$. Using electroplating techniques, copper was electroplated into the molds to form the bottom parts of the coils.

(2) The vertical parts of the coils were fabricated with photolithography technology, too. The vertical parts connecting the bottom parts and the top parts of the coils had a square cross section of $50 \mu \mathrm{m} \times 50 \mu \mathrm{m}$ and a thickness of $20 \mu \mathrm{m}$.

(3) The photoresist layer was removed with acetone and the seed layer was removed by reactive ion etching. 


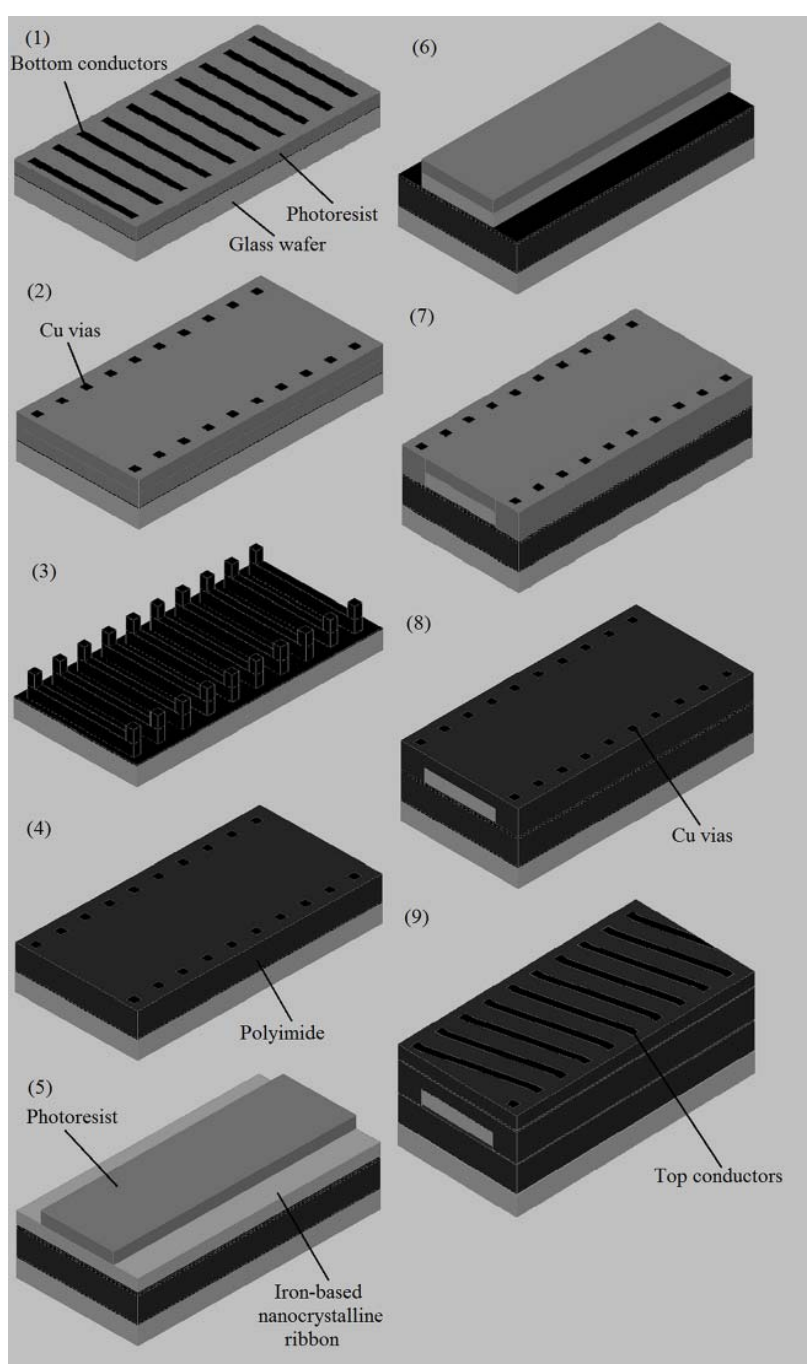

Fig.1. Fabrication steps of the micro fluxgate sensor.

(4) Polyimide with a height just over the vertical vias was spun on the wafer to electrically insulate copper conductors and magnetic core, and to support the sensor structure. Then a polishing process was performed to the polyimide to make the vertical vias exposed and to provide a smooth surface for the following steps.

(5) A titanium protective layer of $300 \mathrm{~nm}$ was deposited on the wafer to protect the finished parts of sensor from being damaged by the following steps. Then a $20 \mu \mathrm{m}$ ironbased nanocrystalline ribbon was glued on the surface of the protective layer with epoxy resin. On the ribbon, a mold of magnetic core was made by lithography.

(6) Using chemical wet etching, the magnetic core was fabricated.

(7) Since the finished vias were not high enough to reach the top parts of the coils fabricated in the later step, the fabrication of vias had to be performed again on the former vias for connecting. So, another seed layer was deposited after removing the epoxy resin and the protective layer. Then, another photoresist layer was spun and patterned on the wafer, and new vias were electroplated right on the previous ones.

(8) Polyimide was spun on the wafer and a polishing process was executed for fabricating the upper layers.
(9) Another seed layer was deposited on the wafer and photoresist molds were prepared with lithography for electroplating the top parts of the coil. After copper was electroplated in the molds, the photoresist was removed and a polyimide process was performed. Then the whole fabrication of fluxgate sensor was finished.

The photograph of the fluxgate sensor is shown in Fig.2. The fluxgate sensor consists of tridimensional solenoid copper coils and a rectangular-ring-shaped magnetic core made of iron-based nanocrystalline alloy. Excitation coils and sensing coil were fabricated by the same processes. The two excitation coils are wound around the two short sides of the rectangular-ring-shaped magnetic core, respectively, and the sensing coil is wound around both the long ones. Thus, the sensing coil is positioned vertically to the excitation coils and between them. Each excitation coil has 18 turns and the sensing coil has 30 turns. The ring width of the magnetic core is $500 \mu \mathrm{m}$. Both the coil line width and the gap between the lines are $50 \mu \mathrm{m}$. Polyimide was used to electrically insulate coils and magnetic core. Moreover, polyimide can support and protect the fluxgate device from vibration and impact. The dimension of the fluxgate sensor was $5.8 \mathrm{~mm} \times 5.5 \mathrm{~mm} \times 120 \mu \mathrm{m}$, including four $1 \mathrm{~mm} \times 1 \mathrm{~mm}$ pads. A new micro fluxgate magnetic sensor has been designed and fabricated.

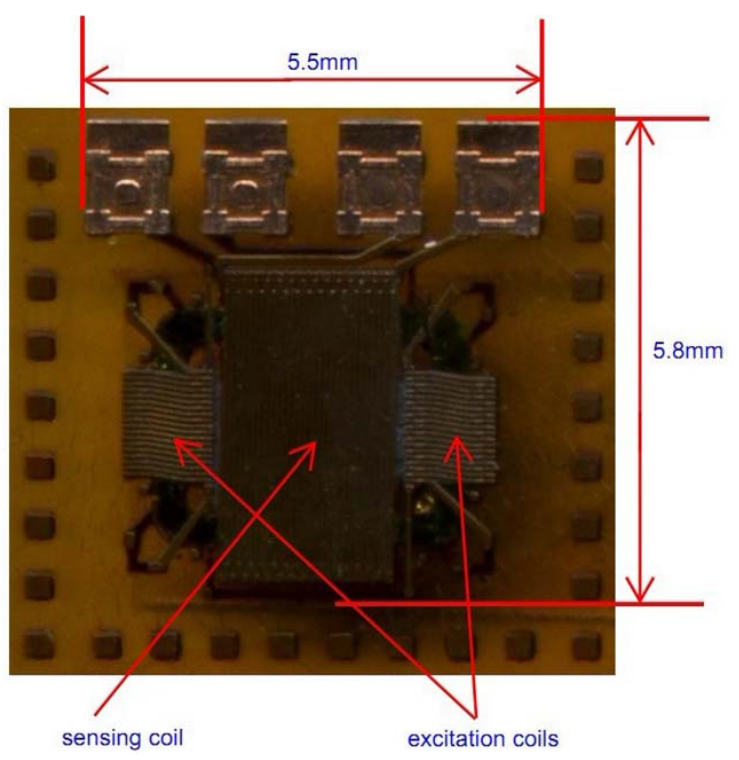

Fig.2. Photograph of the fluxgate sensor.

\section{TESTING SYSTEM AND RESULTS}

Comparing with some other sensors detecting physical properties (pressure, temperature and other) directly, the output signal of magnetic sensors requires some signal processing for translation into the desired parameter [12]. In this work, the fabricated fluxgate sensor was characterized with a magnetic field measuring system based on second harmonic principle. The block diagram of the measuring system is shown in Fig.3.

Generally, reducing the size of fluxgate sensor often causes a decrease in sensitivity. An applicable solution that can compensate this drawback is to increase the operational frequency. It means that more complicated supporting 
electronic circuits are required to process the excitation and output signals. For the reasons above, the function generator was set to provide sine wave signals with a frequency of 100 $\mathrm{kHz}$ in this work.

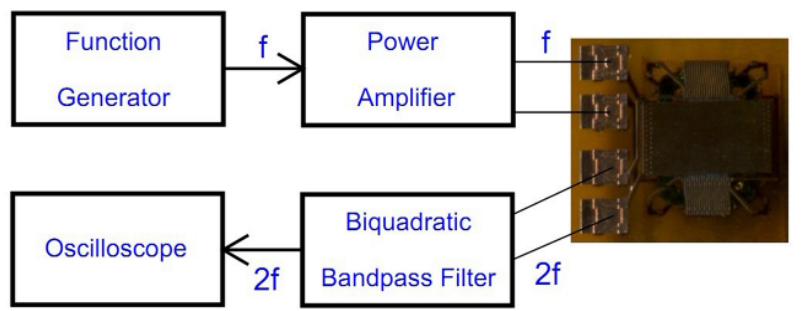

Fig.3. Block diagram of the measuring system.

To ensure that the magnetic core was working in deep saturation state, a power amplifier connecting the function generator and the excitation coils was used to provide powerful enough currents. In these experiments, excitation currents with different effective values were provided to characterize the fluxgate sensor.

In this measuring system, when $100 \mathrm{kHz}$ sine waveform excitation signal was selected to drive the excitation coils, $200 \mathrm{kHz}$ sine waveform signal generated by the sensing coil should be picked up as output signal indicating the intensity of the magnetic field. Unfortunately, accompanying the useful signal were always some signals with other frequencies generated by the sensing coil. To obtain the accurate measuring results, a signal processing circuit being able to distinguish the $200 \mathrm{kHz}$ signal from those with other frequencies was needed to process the output signals of the sensing coil. So, a band pass filter with a center frequency of $200 \mathrm{kHz}$ was used to process the output signals of the sensing coil.

The input of the band pass filter was connected with the sensing coil of the fluxgate sensor. Thus, the output signals of the band pass filter acting as final experiment results were read by the oscilloscope.

The magnetic field under test was produced by a copper solenoid with 800 turns and with a length of $0.15 \mathrm{~m}$. The DC current through the solenoid was controlled by a DC power supply. To remove the interference of the earth magnetic field, the solenoid and the sensor were placed in a cylindraceous shield consisting of three layers of Metglas 2714A ribbon with a thickness of $20 \mu \mathrm{m}$. Moreover, the shield was placed with its axis vertical to the earth magnetic field. The micro fluxgate sensor was placed in the solenoid with the axis of its sensing coil parallel to the DC magnetic direction produced by the solenoid. Fig. 4 shows the output signals of the fluxgate sensor with/without external magnetic field.

Fig.5 shows the curves representing the relationship between the output signals of the fluxgate sensor and the external magnetic field with different excitation currents.

In Fig.5, some asymmetry can be found in the curves, which indicates that there was a small difference between the sensitivity characteristics and the sensor measuring positive or negative DC magnetic field. This difference might be caused by the undesirable imperfect symmetric structure of the sensor.

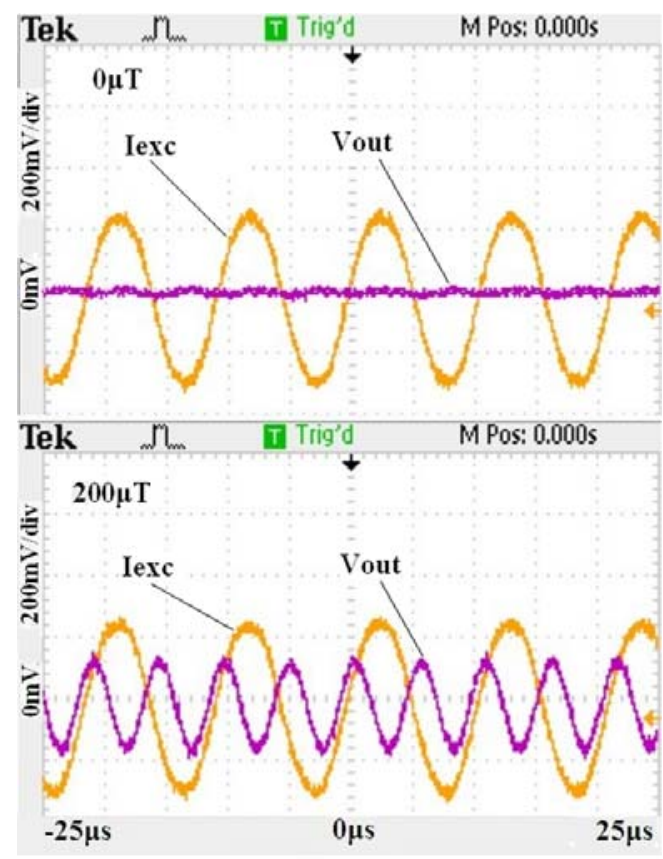

Fig.4. Output signals of the fluxgate sensor with/without external magnetic field

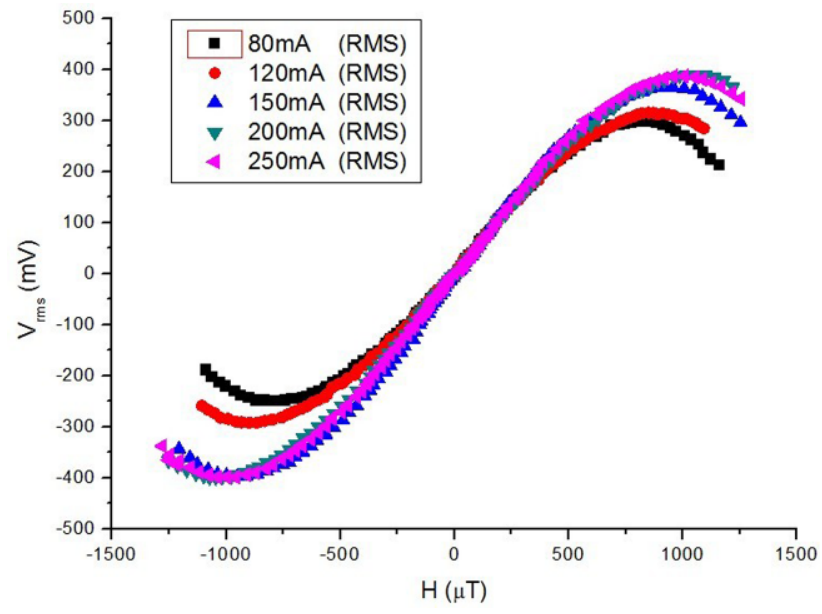

Fig.5. (a) Output signals of the fluxgate sensor vs. external magnetic field

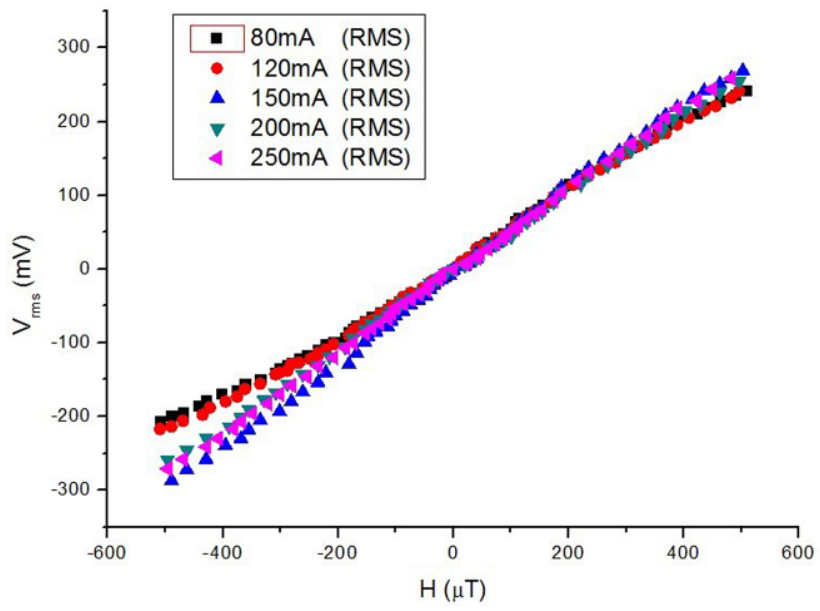

Fig.5. (b) Output signals of the fluxgate sensor vs. external magnetic field in the linear range 
When the excitation signal was the sine wave with a frequency of $100 \mathrm{kHz}$, the fabricated fluxgate sensor was tested with the RMS excitation currents of $80 \mathrm{~mA}, 120 \mathrm{~mA}$, $150 \mathrm{~mA}, 200 \mathrm{~mA}$ and $250 \mathrm{~mA}$, respectively. According to the experiment results, the maximum sensitivity was achieved when the excitation current was $150 \mathrm{~mA}$. Through calculation, the linear range was $-500 \mu \mathrm{T}$ to $+500 \mu \mathrm{T}$ and the maximum sensitivity was $583.1 \mathrm{~V} / \mathrm{T}$ when the total amplification gain of the signal processing circuit was 6 . The sensor noise was measured for the sine waveform excitation signals, $\mathrm{f}_{\mathrm{exc}}=100 \mathrm{kHz}, \mathrm{I}_{\mathrm{exc}}=150 \mathrm{~mA}$ RMS. The noise power density was $13.57 \mathrm{nT} / \mathrm{Hz}^{0.5}$ at $1 \mathrm{~Hz}$ and the noise RMS level was $23 \mathrm{nT}$ in the frequency range of $10 \mathrm{mHz}-25$ $\mathrm{Hz}$. The resistance of excitation coils was around $1.5 \Omega$, so power consumption was $33.75 \mathrm{~mW}$ with the excitation current of $150 \mathrm{~mA}$.

\section{Conclusions}

This paper presents a fluxgate magnetic sensor with tridimensional micro-solenoid coils and a rectangular-ringshaped magnetic core made of iron-based nanocrystalline alloy. Using thick photoresist-based UV lithography and electroplating, the tridimensional solenoid coils were fabricated. Gluing and chemical wet etching was adopted to process the nanocrystalline alloy magnetic core. Polyimide was used to electrically insulate coils and magnetic core, and to protect the structure of the fluxgate sensor from vibration and impact. When the excitation frequency was $100 \mathrm{kHz}$ and the effective excitation current was $150 \mathrm{~mA}$, the maximum sensitivity of $583.1 \mathrm{~V} / \mathrm{T}$ was achieved in the linear range from $-500 \mu \mathrm{T}$ to $+500 \mu \mathrm{T}$. Using the fluxgate sensor with high sensitivity, wide measuring range, lower noise, small size and low power consumption, numerous applications are expected, such as GPS positioning equipment, navigation system, and space research.

\section{ACKNOWLEDGMENT}

This work was supported by The National Natural Science Foundation of China (No. 61074168 and No. 61273065), National Science and Technology Support Program (2012BAK08B05) and National Key Laboratory Research Fund (9140C790403110C7905).

\section{REFERENCES}

[1] Liakopoulos, T.M., Ahn, C.H. (1999). A microfluxgate magnetic sensor using micromachined planar solenoid coils. Sensors and Actuators A: Physical, 77, 66-72.

[2] Primdahl, F. (1979). The fluxgate magnetometer. Journal of Physics E: Scientific Instruments, 12, 241-253.

[3] Lenz, J.E. (1990). A review of magnetic sensors. Proceedings of the IEEE, 78 (6), 973-989.

[4] Ripka, P. (2000). New directions in fluxgate sensors. Journal of Magnetism and Magnetic Materials, 215216, 735-739.

[5] Popovic, R.S., Flanagan, J.A., Besse, P.A. (1996). The future of magnetic sensors. Sensors and Actuators A: Physical, 56, 799-802.

[6] Ripka, P. (2003). Advances in fluxgate sensors. Sensors and Actuators A: Physical, 106, 8-14.

[7] Acuna, M.H. (2002). Space-based magnetometers. Review of Scientific Instruments, 73 (11), 3717-3736.

[8] Kejik, P., Chiesi, L., Janossy, B., Popovic, R.S. (2000). A new compact 2D planar fluxgate sensor with amorphous metal core. Sensors and Actuators A: Physical, 81, 181-183.

[9] Zorlu, O., Kejik, P., Popovic, R.S. (2007). An orthogonal fluxgate-type magnetic microsensor with electroplated permalloy core. Sensors and Actuators A: Physical, 135, 43-49.

[10] Kawahito, S., Satoh, H., Sutoh, M., Todokoro, Y. (1996). High resolution micro fluxgate sensing elements using closely coupled coils structures. Sensors and Actuators A: Physical, 54, 612-617.

[11] Koch, R.H., Rozen, J.R. (2001). Low-noise flux-gate magnetic-filed sensors using ring- and rod-core geometries. Applied Physics Letters, 78, 1897-1899.

[12] Laurinavičius, L. (2011). Helicon resonator based strong magnetic field sensor. Measurement Science Review, 11, 149-153. 